https://doi.org/10.22319/rmcp.v11s2.4694

Artículo

\title{
Efecto en la erosión hídrica del suelo en pastizales y otros tipos de vegetación por cambios en el patrón de lluvias por el calentamiento global en Zacatecas, México
}

Francisco Guadalupe Echavarría-Cháirez ${ }^{\mathrm{a}^{*}}$

Guillermo Medina-García a

José Ariel Ruiz-Corral ${ }^{\mathrm{b}}$

a Instituto Nacional de Investigaciones Forestales Agrícolas y Pecuarias (INIFAP). Campo Experimental Zacatecas, Km 24.5 Carretera Zacatecas-Fresnillo, Calera, Zac., México.

${ }^{\mathrm{b}}$ Universidad de Guadalajara. Centro Universitario de Ciencias Biológicas y Agropecuarias. Camino Ramón Padilla Sánchez No. 2100 Nextipac, 44600, Zapopan, Jalisco, México.

*Autor de correspondencia: echavarria.francisco@inifap.gob.mx.

\section{Resumen:}

Para evaluar la erosión hídrica se utilizaron anomalías de temperatura y precipitación para el período 2021-2080, estimadas con un ensamble de 11 modelos de circulación global, para generar mapas de temperatura y precipitación futuras a partir de la climatología de referencia 1961-2010. En el caso del estado de Zacatecas el área de pastizal presenta valores promedio actuales de erosión del orden de 16.3 t/ha, estimadas mediante el modelo RUSLE. En otros tipos de vegetación o usos del suelo como es el matorral, bosque o el área agrícola se generan valores aún mayores (50.74, 65.99, 161.39, t/ha/año, respectivamente). La aplicación del modelo ensamble en las RCP 4.5 y RCP 8.5 indica que habrá un incremento en la temperatura y una disminución de la lluvia. En un escenario de menor efecto de calentamiento global (RCP 4.5) se espera una reducción gradual de erosión hídrica del pastizal respecto a 2010 de $2.3 \%$ en 2030 (15.67 t/ha/año) hasta un 5.88\% ( 15.18 t/ha/año) en 2070. En el mismo periodo hasta 2070, la tasa de decremento de erosión hídrica es de $14.1 \mathrm{~kg} / \mathrm{ha} / \mathrm{año}$. La RCP 8.5, que fue diseñada para una condición de continua emisión de gases de invernadero, presenta valores de reducción aún mayores y para 2070 establece una 
disminución del $13.0 \%$, con una tasa de disminución de erosión de $31.5 \mathrm{~kg} / \mathrm{ha} /$ año. Otros tipos de vegetación y uso del suelo mostraron la misma tendencia de disminución, aunque en tasas de decremento más altas. Aunque el escenario de disminución de erosión hídrica parece favorable, no se descarta un incremento de valores de pérdida de suelo por efecto de erosión eólica en las regiones áridas del norte del estado de Zacatecas. La valoración de niveles de erosión hídrica contribuye al ordenamiento del uso del suelo para reducir niveles de degradación y desertificación.

Palabras clave: Erosión hídrica, RUSLE, Cambio climático, RCP.

Recibido: $12 / 11 / 2017$

Aceptado: 22/08/2018

\section{Introducción}

La erosión hídrica del suelo es solo uno de los tipos de erosión posibles y una de las formas de degradación del suelo que existen. En particular, la erosión hídrica es un proceso continuo que afecta los suelos de todo el planeta. Los cambios en la cantidad, intensidad y distribución de la lluvia aceleran o reducen el efecto continuo de la erosión. Esos cambios se están presentando actualmente como consecuencia del incremento en los gases de efecto invernadero (GEI), que influyen en el calentamiento global ${ }^{(1)}$ y el cambio climático.

Las pérdidas de suelo por erosión hídrica son de mayor importancia, especialmente en sitios donde la capa superficial es poco profunda, tal es el caso del estado de Zacatecas. Los suelos de pastizal se caracterizan por su reducida profundidad, en cambio los suelos de uso agrícola son de mayor profundidad. El efecto de pérdidas de suelo será diferente en función del uso del mismo, pero en todos los casos, conducirá a la reducción paulatina de la productividad y degradación.

Existe gran incertidumbre sobre los efectos que el cambio climático provocará en las actividades productivas, esto debido a que dichas actividades y en consecuencia la seguridad alimentaria, dependen de las precipitaciones pluviales. Sin embargo, indicadores como la erosión hídrica pudieran no ser del interés general, sobre todo, aquellos indicadores que no son medidos de manera rutinaria y cuyos efectos, por presentarse permanentemente, no son fácilmente perceptibles. El conocimiento actual y futuro de las posibles pérdidas de suelo y la degradación en general, son de importancia para la planeación de uso del suelo y su ordenamiento ${ }^{(2)}$. La Sociedad Americana de la Ciencia del Suelo en su glosario ${ }^{(3)}$ define la degradación como: "el proceso a través del cual un compuesto es transformado en componentes más simples". La desertificación fue definida en 1992 por la Convención de las Naciones Unidas de Lucha Contra la Desertificación como: "la degradación de las tierras de zonas áridas, semiáridas y subhúmedas secas, resultante de diversos 
factores, tales como las variaciones climáticas y las actividades humanas"(4). La CONABIO(5) menciona que en 2011, la superficie de ecosistemas naturales del país se ha reducido significativamente desde mediados del siglo pasado para transformarse en terrenos agropecuarios, zonas urbanas y obras de infraestructura, que el $28.7 \%$ del territorio había perdido sus ecosistemas naturales y el restante 71.3 \% los mantenía con diferentes grados de conservación. Por lo anterior, la planeación y ordenamiento del uso del suelo, son fundamentales para mantener estables los niveles de degradación y la desertificación a través del mantenimiento de la frontera agrícola y de reducir al máximo los cambios de uso del suelo. En este sentido, es importante evaluar el efecto del cambio climático sobre los niveles de erosión hídrica en las áreas de pastizal y otros tipos de vegetación, para contribuir a la planeación de acciones de protección, al ordenamiento ecológico y reducción de la degradación del suelo.

\section{Material y métodos}

El estado de Zacatecas se localiza entre las coordenadas $25^{\circ} 07^{\prime} 32^{\prime \prime} \mathrm{N}$ y $21^{\circ} 01^{\prime} 48^{\prime \prime} \mathrm{N}$ y entre los $100^{\circ} 44^{\prime} 09^{\prime \prime}$ y $104^{\circ} 24^{\prime} 08^{\prime \prime}$ O y cuenta con una extensión de $7^{\prime} 447,970.8$ ha $^{(6)}$. El tipo de vegetación predominante en el estado de Zacatecas es el matorral xerófilo que ocupa 3’173,280 ha, seguido por la superficie ocupada por el área agrícola de 1`746,987 ha, después la superficie ocupada por el pastizal que representa una superficie de $1^{\prime} 454,234$ ha y en cuarto lugar la superficie de bosque que ocupa 1'125,285 ha ${ }^{(7)}$; el resto lo ocupan selva y otros tipos de vegetación de menor dimensión. Los suelos predominantes en el estado son 14 unidades, de las que sobresalen Xerosol (38.8 \%), Litosol (14.3\%), Feozem (14.0\%), Regosol (12.2\%) y Castañozem $(9.6 \%)^{(8)}$.

\section{Erosión hídrica}

Para estimar la erosión hídrica actual, se utilizó la Ecuación Universal de Pérdida de Suelo Revisada (RUSLE por sus siglas en inglés) ${ }^{(9)}$ :

$$
\mathrm{E}=\mathrm{R} * \mathrm{~K} * \mathrm{~L} * \mathrm{~S} * \mathrm{C} * \mathrm{P}
$$

\section{Donde:}

$\mathbf{E}=$ pérdida de suelo por unidad de área (t/ha/año);

$\mathbf{R}=$ factor de erosividad de la lluvia $\left(\mathrm{MJ} \mathrm{mm} \mathrm{ha}^{-1} \mathrm{~h}^{-1} \mathrm{año}^{-1}\right)$;

$\mathbf{K}=$ factor de erodabilidad del suelo $\left(\mathrm{t} \mathrm{ha}^{-1} \mathrm{~h}^{-1} / \mathrm{MJ} \mathrm{mm} \mathrm{ha}^{-1}\right)$;

$\mathbf{L}=$ factor de longitud de la pendiente (adimensional);

$\mathbf{S}=$ factor de grado de la pendiente (adimensional);

$\mathbf{C}=$ factor de la vegetación (adimensional);

$\mathbf{P}=$ factor de práctica mecánica de control de la erosión (adimensional). 
Para su medición se utilizó el programa IDRISI selva ${ }^{(10)}$, el cual incluye el comando RUSLE, que es una rutina que mejora el cálculo de los factores $\mathrm{L}$ y $\mathrm{S}$ e incluyendo otras rutinas adicionales que elimina depresiones, y otros que permiten una generalización mejor del modelo digital de elevación. Para la estimación del factor $\mathrm{R}$ se usó el mapa de isoerosividad, que divide al país en 14 regiones, de las cuales, el estado de Zacatecas se ubica en las regiones 3, 4 y 7 y cuyas ecuaciones son las siguientes:

$$
\begin{aligned}
& Y=3.67516 x-0.001720 X^{2}(2) \\
& Y=2.89594 x+0.002983 X^{2}(3) \\
& Y=0.03338 x+0.006661 X^{2}(4)
\end{aligned}
$$

En donde $\mathrm{Y}$ es el factor $\mathrm{R}$ en términos de $\mathrm{MJ} \mathrm{mm} / \mathrm{ha} \mathrm{h}$ y $\mathrm{X}$ es la lluvia anual en $\mathrm{mm}$.

Para la estimación de los valores del parámetro K, se utilizó una tabla de valores de erosionabilidad desarrollada por Figueroa ${ }^{(11)}$. El factor K está en función de la textura y clase de suelo. Estos valores se asignaron a las clases de suelo y texturas dentro de los terrenos ejidales, las cuales se digitalizaron para facilitar su manejo dentro de los sistemas de información geográfica (SIG), por medio del programa IDRISI Selva ${ }^{(10)}$. La salida es la pérdida de suelo para cada tipo de vegetación.

Para cuantificar el efecto del cambio climático se utilizó el sistema de información de cambio climático de la República Mexicana del INIFAP ${ }^{(12)}$, el cual consta de climatología base del periodo 1961-2010 y climatología de los años 2021 a 2080 en las rutas de concentración representativas (RCP) 4.5 y 8.5 de gases de efecto invernadero (GEI), entre otros para un modelo ensamble formado a partir de 11 modelos de circulación general (GCM) reducidos en escala y calibrados ${ }^{(13)}$ seleccionados para México (BCC- CSM1- 1, CCSM4, GISS- E2- R, HadGEM2- AO, HadGEM2- ES, IPSLCM5A- LR, MIROC- ESM- CHEM, MIROC- ESM, MIROC5, MRICGCM3, NorESM1- M).

Se utilizaron los valores de temperatura máxima, temperatura mínima y precipitación mensuales para los años 2021 a 2080. Con los datos mensuales se obtuvieron los anuales para los escenarios climáticos 2021-2040, 2041-2060 y 2061-2080, en adelante referidos como climatologías o años 2030, 2050 y 2070, respectivamente.

Utilizando la información de uso del suelo de INEGI ${ }^{(14)}$, fue posible separar los efectos de disminución de lluvia y erosión hídrica por tipo de vegetación predominante. Adicional al área de pastizal, se utilizó la información de la superficie dedicada a otros tipos de vegetación como son el matorral, bosque y el área de uso agrícola. Con la información de erosión hídrica estimada para la actualidad y la de los años 2030, 2050 y 2070 se obtuvieron modelos de regresión ${ }^{(15)}$ para los tipos de vegetación más importantes del Estado, ya mencionadas, obteniéndose una tasa anual de cambio para cada condición de vegetación y con ello comparar contra el área de pastizal y determinar donde se obtendrán los mayores impactos por efecto del cambio climático. 


\section{Resultados y discusión}

Contrario a lo que sucede con otros indicadores de cambio, en lo que respecta a las pérdidas de suelo por efecto de la erosión hídrica, ésta tenderá a disminuir a medida que el efecto de calentamiento global, provoque una disminución de la precipitación pluvial en el estado de Zacatecas.

El Cuadro 1 presenta las superficies susceptibles de erosión hídrica y el rango de pérdida de suelo asociada a cada una de ellas, tanto en la climatología de referencia como en las climatologías y escenarios de cambio climático estudiados. Los escenarios utilizados para proyectar las precipitaciones que se utilizaron para el cálculo de valores de erosión hídrica, son los denominados como rutas representativas de concentración de gases efecto invernadero (RCP) 4.5 y 8.5, para las climatologías 2030, 2050 y 2070. Las RCP se caracterizan por el cálculo aproximado que hacen del forzamiento radiativo total en el año 2100 en relación con el año 1750 (IPCC, 2013), esto es $4.5 \mathrm{~W}$ m-2, en el caso del escenario RCP4.5 (emisiones intermedias de GEI) y $8.5 \mathrm{Wm}-2$ en el caso del escenario RCP8.5 (emisiones altas de GEI).

Cuadro 1: Superficies (ha) de pérdida de suelo asociada a pastizales, estimadas para el RCP 4.5 con el modelo RUSLE para el estado de Zacatecas desde el año 2010 hasta 2070

\begin{tabular}{|c|c|c|c|c|c|}
\hline \multirow{2}{*}{$\begin{array}{c}\text { Erosión } \\
\text { (t/ha) }\end{array}$} & \multicolumn{4}{|c|}{ Años } & \multirow{2}{*}{ Diferencia (\%) } \\
\cline { 2 - 5 } & 2010 & 2030 & 2050 & 2070 & 2.57 \\
\hline $0-10$ & 888,417 & 894,767 & 906,833 & 911,278 & -3.57 \\
\hline $10-100$ & 533,431 & 529,621 & 519,460 & 514,380 & -12.50 \\
\hline $100-500$ & 30,482 & 27,942 & 26,036 & 26,671 & \\
\hline
\end{tabular}

En el Cuadro 2 se presentan las proyecciones de superficies asociadas al rango de pérdida de suelo bajo la RCP 8.5, que es la condición en la que no se espera ninguna disminución del efecto invernadero. La Figura 1, muestra los cambios en erosión hídrica en las áreas de pastizal del estado de Zacatecas, con los RCP 4.5 y 8.5 .

Cuadro 2: Superficies (ha) de pérdida de suelo asociada a pastizales, estimadas para el RCP 8.5 con el modelo RUSLE para el estado de Zacatecas desde el año 2010 hasta 2070

\begin{tabular}{|c|c|c|c|c|c|}
\hline \multirow{2}{*}{$\begin{array}{c}\text { Erosión } \\
\text { (t/ha) }\end{array}$} & \multicolumn{4}{|c|}{ Años } & \multirow{2}{*}{ Diferencia (\%) } \\
\cline { 2 - 5 } & 2010 & 2030 & 2050 & 2070 & 5.72 \\
\hline $0-10$ & 888,417 & 910,643 & 930,329 & 939,220 & -7.38 \\
\hline $10-100$ & 533,431 & 515,015 & 502,949 & 494,059 & -37.50 \\
\hline $100-500$ & 30,482 & 26,671 & 19,051 & 19,051 & \\
\hline
\end{tabular}


Figura 1: Erosión hídrica actual (izq.) y proyectada a 2070 (der. sup) con RCP 4.5 y RCP 8.5 (der. inf.) del pastizal en el estado de Zacatecas
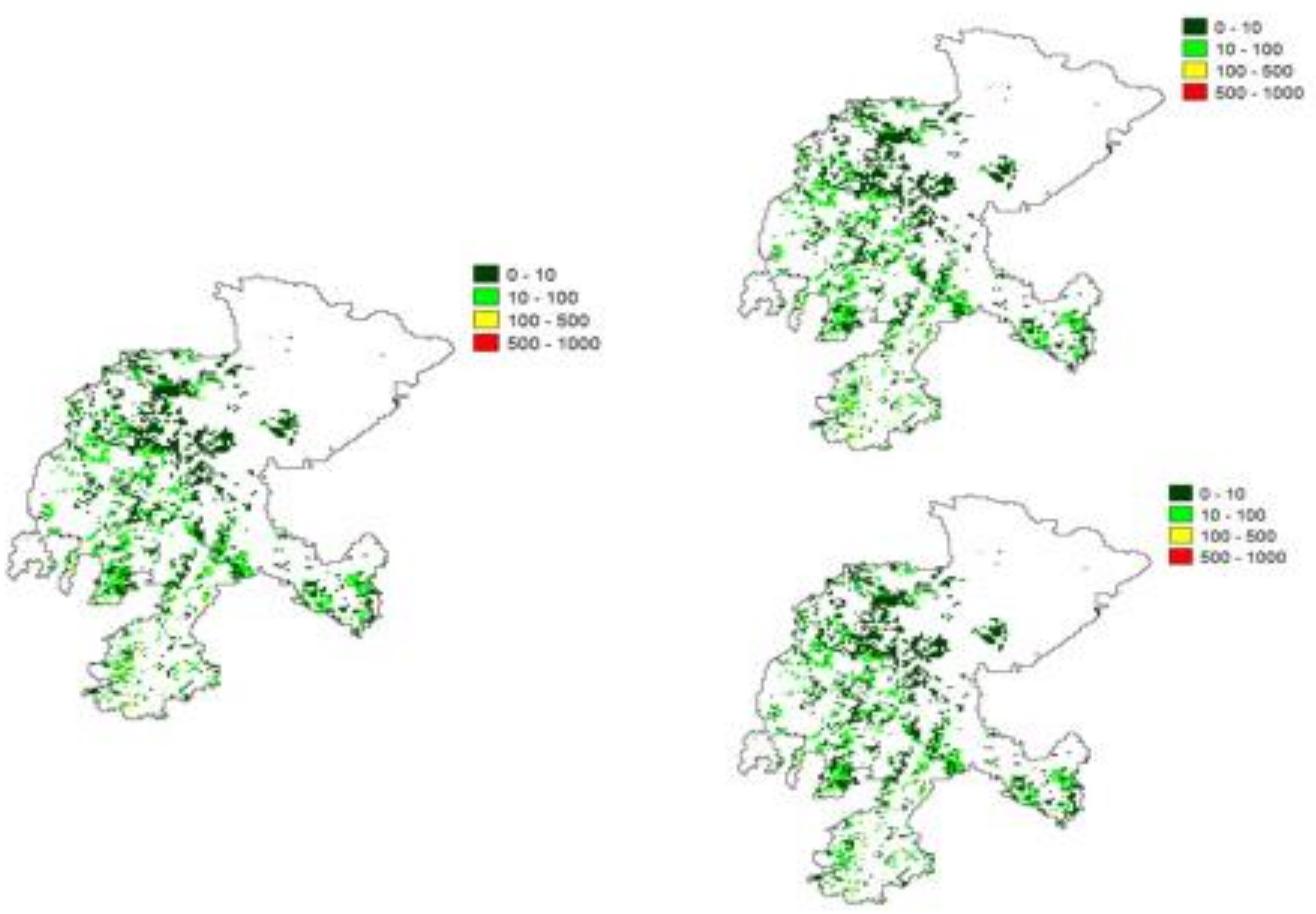

En ambos Cuadros (1 y 2) las cifras muestran una tendencia semejante, la categoría que representa la mayor superficie en ambos cuadros, es la asociada al rango de erosión de 0 a 10 t/ha, la cual representa un poco más del $60 \%$ del total de la superficie de pastizal en el estado de Zacatecas. En ambos RCP dicha categoría tiende a incrementarse. Con el RCP 4.5 se incrementa en 22,861 ha $(2.57 \%)$ pero con el RCP 8.5, el incremento es de 50,803 ha, lo que representa aproximadamente el doble $(5.72 \%)$ de la superficie estimada por el RCP 4.5, esto para el año 2070.

Dado que en ambos RCP se proyectó una disminución en la precipitación de 4.0 y $7.8 \%$ (Cuadros 3 y 4) para RCP4.5 y RCP8.5, respectivamente, hasta el año 2070, lo cual parece una contradicción, disminuye la precipitación y aumenta la erosión. Sin embargo, en las categorías de los valores altos de erosión hídrica, en los que se encuentran los valores de 10 a 500 t/ha, muestran que, en esos niveles de mayor erosión, es donde se presentará una estabilidad, incluso una disminución. Aunque los valores de las categorías 10-100 y 100-500, corresponden con los valores menores en superficie (40\%), son en estas categorías donde se presentan los valores más altos de erosión hídrica, por lo que la estabilidad y disminución en ellas, conduce a una disminución en los valores medios totales para la superficie de pastizal. Al utilizar los valores medios de erosión para el estado de Zacatecas, en un análisis de regresión, se encontraron tasas de cambio negativas y en 
ambos casos significativas $(P<0.05)$, con valores pequeños de reducción por año en los RCP 4.5 (14.1 kg/ha/año) y RCP 8.5 (31.5 kg/ha/año (Figura 2).

Cuadro 3: Precipitación media proyectada mediante RCP 4.5 y porcentaje de reducción en el periodo de 2010 a 2070

\begin{tabular}{|l|r|r|r|r|r|}
\hline \multirow{2}{*}{ Uso del suelo } & \multicolumn{4}{|c|}{ Años } & \multirow{2}{*}{\begin{tabular}{c} 
Reducción \\
\cline { 2 - 5 }
\end{tabular}} \\
\cline { 1 - 5 } Pastizal & 2010 & 2030 & 2050 & 2070 & $(\%)$ \\
\hline Matorral & 500 & 492 & 483 & 480 & 4.04 \\
\hline Agrícola & 385 & 377 & 370 & 367 & 4.53 \\
\hline Bosque & 469 & 462 & 454 & 450 & 4.03 \\
\hline
\end{tabular}

Cuadro 4: Precipitación media proyectada mediante RCP 8.5 y porcentaje de reducción en el periodo de 2010 a 2070

\begin{tabular}{|l|r|r|r|r|r|}
\hline \multirow{2}{*}{ Uso del suelo } & \multicolumn{4}{|c|}{ Años } & Reducción \\
\cline { 1 - 5 } Pastizal & 2010 & 2030 & 2050 & 2070 & $(\%)$ \\
\hline Matorral & 500 & 482 & 464 & 461 & 7.87 \\
\hline Agrícola & 385 & 369 & 353 & 351 & 8.81 \\
\hline Bosque & 469 & 452 & 436 & 432 & 7.88 \\
\hline
\end{tabular}

Figura 2: Pérdidas promedio anuales de suelo en pastizal del estado de Zacatecas con la RCP 4.5 (izquierda) y RCP 8.5 (derecha)
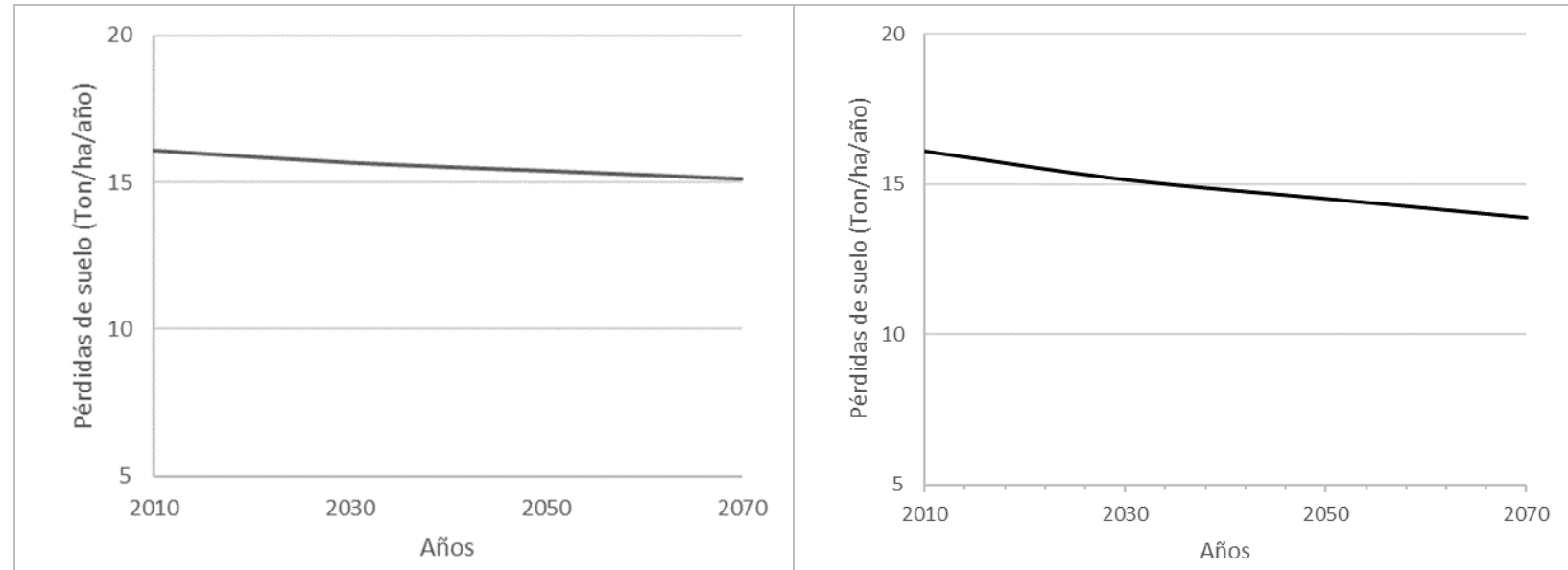


\section{Comparación con otros tipos de vegetación}

El efecto de la disminución de precipitación en la reducción de erosión hídrica del suelo del pastizal en Zacatecas es diferente para otros tipos de vegetación en el Estado; al evaluar el impacto en la reducción de lluvia en las áreas de matorral, bosque y de uso agrícola, los valores estimados para el área de matorral son los menores, (Cuadro 3), aunque se observa un patrón semejante al proyectado para el área de pastizal. El Cuadro 3 presenta los valores de precipitación estimados para cada tipo de vegetación y la reducción en porcentaje. Los valores de reducción son menores para el RCP 4.5 que para el RCP 8.5, debido a que el primero se asocia con un menor efecto de los gases de efecto invernadero (GEI). La reducción en los valores de precipitación afecta los valores medios de erosión de los diversos tipos de vegetación estudiados.

En los Cuadros 5 y 6 se puede observar la reducción en los valores medios de erosión hídrica. estimados mediante el RCP 4.5 y el RCP 8.5. En el Cuadro 5 se observa una disminución en porcentaje de entre 5 y $9 \%$ de los valores actuales (2010), en el que sobresale el área de pastizal cuyos valores van de 16.13 a $15.18 \mathrm{t} / \mathrm{ha} / \mathrm{año}$, lo que representa un $5.89 \%$ de reducción en 60 años y mostrando los valores más bajos de todos los tipos de vegetación. Aunque esta proyección puede ser alterada por cambios en el uso del suelo. En esta área se realiza el pastoreo y está sujeta a riesgos de deterioro, sobre todo cuando se presenta el sobrepastoreo $^{(16)}$, el cual se reconoce como una de las principales causas de incremento de escurrimiento y erosión hídrica, ya que, al reducirse la cobertura vegetal, se reduce la infiltración y aumenta el escurrimiento ${ }^{(17)}$. La disminución de la cobertura vegetal también puede ir asociada a la reducción de precipitación. Periodos largos de sequía han sido reconocidos como causantes y coadyuvantes en el incremento de erosión. El riesgo que corre el pastizal por el cambio de uso de suelo, ha sido mostrado en los reportes de tasas de cambio de uso de suelo en su conversión hacia áreas de agricultura de riego, durante los años setentas del siglo pasado, en la que, anteriormente era el área de pastizal dentro del acuífero denominado "Chupaderos" dentro del estado de Zacatecas, las cuales fueron de hasta 1,913 ha/año y de 678 ha/año en el acuífero del Aguanaval ${ }^{(18)}$. Un cambio en el uso de suelo puede inducir cambios en la erosión hídrica de un área de pastizal a valores de erosión propios del área agrícola, cuyos valores medios van desde 133 hasta 161 t/ha/año. 
Cuadro 5: Valores medios de erosión hídrica (t/ha/año) por tipo de vegetación con precipitaciones proyectadas mediante RCP 4.5

\begin{tabular}{|l|c|c|c|c|c|}
\hline \multirow{2}{*}{$\begin{array}{c}\text { Tipos de } \\
\text { vegetación }\end{array}$} & \multicolumn{4}{|c|}{ Años } & Diferencia \\
\cline { 2 - 5 } & 2010 & 2030 & 2050 & 2070 & $(\%)$ \\
\hline Pastizal & 16.13 & 15.67 & 15.29 & 15.18 & -5.89 \\
\hline Matorral & 50.74 & 49.76 & 48.62 & 48.11 & -5.18 \\
\hline Bosque & 65.99 & 64.47 & 61.24 & 61.46 & -6.86 \\
\hline Agrícola & 161.39 & 157.32 & 146.35 & 145.56 & -9.81 \\
\hline
\end{tabular}

Cuadro 6: Valores medios de erosión hídrica (t/ha/año) por tipo de vegetación con precipitaciones proyectadas mediante RCP 8.5

\begin{tabular}{|l|c|c|c|c|c|}
\hline \multirow{2}{*}{$\begin{array}{c}\text { Tipos de } \\
\text { vegetación }\end{array}$} & \multicolumn{4}{|c|}{ Años } & Diferencia \\
\cline { 2 - 5 } & 2010 & 2030 & 2050 & 2070 & $(\%)$ \\
\hline Pastizal & 16.13 & 15.19 & 14.28 & 14.03 & -13.02 \\
\hline Matorral & 50.74 & 47.94 & 45.68 & 45.01 & -11.29 \\
\hline Bosque & 65.99 & 61.12 & 64.47 & 54.51 & -17.40 \\
\hline Agrícola & 161.39 & 151.2 & 142.32 & 133.18 & -17.48 \\
\hline
\end{tabular}

De igual manera se aprecian valores medios de erosión hídrica que disminuirán en el área de matorral y que van desde el $5.18 \%$, hasta el $11.29 \%$, esto debido a la disminución de lluvia pronosticada para los próximos años y cuyas tasas de cambio son significantes $(P<0.05)$. Al igual que el pastizal, el sobrepastoreo y el cambio de uso de suelo son los mayores riesgos de que esta disminución de erosión hídrica no sea tal como se pronostica. Otro riesgo para esta área es la erosión eólica, ya que es en este tipo de vegetación donde se presentarán las menores precipitaciones de todo el Estado, lo que puede favorecer el incremento en la erosión eólica asociada a la sequía ${ }^{(19,20)}$. Una situación semejante a las dos anteriores, se observará en el área de bosque, donde se pronostican reducciones de erosión hídrica de 6.86 hasta $17.4 \%$, sin que las tasas de cambio sean significativas $(P>0.05)$. En el caso de este tipo de vegetación, la tala de árboles o cambio de suelo se aprecian como los mayores riesgos, así como el efecto de la sequía y enfermedades en los árboles, los cuales se han ido presentando recientemente ${ }^{(21)}$.

Por último, se menciona el caso del área agrícola, la cual por ubicarse casi siempre en la parte más baja de la cuenca, está sujeta a escurrimientos de mayor magnitud y mayor erosión. También son los sitios de mayor profundidad de suelo, lo que la vuelve el área de mayor cantidad de pérdidas de suelo. Sin embargo, ante la perspectiva de disminución de precipitación, se estima que los valores de erosión hídrica se reducirán de un 9.81 a un $17.48 \%$, siendo esta área la más beneficiada en términos de disminución de erosión hídrica, con tasas de cambio menores $(\mathrm{P}>0.05)$. El manejo apropiado del recurso suelo, manejo de pendientes, técnicas de labranza de conservación del suelo y el mantenimiento de la cobertura vegetal, son componentes tecnológicos que contribuyen a mejorar el cuidado del suelo. Sin embargo, las prácticas de manejo del suelo tradicionales, surcos 
a favor de la pendiente y el laboreo en periodos de vientos intensos, pueden incrementar los valores de erosión hídrica y eólica. Esta última ha sido reportada ${ }^{(11)}$ como un riesgo de erosión equivalente a la que provoca el escurrimiento. El establecimiento de barreras rompe viento y cambios en las técnicas de laboreo y conservación de humedad, deberán ser prioritarias para mantener la productividad agrícola y no incrementar los valores de erosión hídrica, tal como lo pronostican los modelos usados aquí. Adicionalmente, se debe poner atención a la reducción de autorizaciones de cambio de uso del suelo, ya que como se mencionó, el cambio de uso del suelo puede llevar a los niveles de pérdida de suelo equivalentes a los que se presentan en el área agrícola.

Por otro lado, aunque parece que los efectos más notorios de las pérdidas de suelo en Zacatecas, se presentan en el área agrícola, las pérdidas de suelo en otros tipos de vegetación son tanto o más perjudiciales que en el área agrícola, debido a que la profundidad de suelo en las áreas de pastizal, bosque o matorral, son mucho menores que en las superficies de uso agrícola. Por lo tanto, una disminución de un centímetro de capa superficial de suelo, provoca efectos más dramáticos en esas áreas que en las agrícolas.

Como se mencionó al inicio de esta sección, que a diferencia de lo que sucede con otros indicadores, lo que respecta a las pérdidas de suelo por efecto de la erosión hídrica, ésta tenderá a disminuir a medida que el efecto de calentamiento global debido a la acumulación de gases en la atmósfera, provoquen una disminución de la precipitación pluvial en el estado de Zacatecas. Los resultados muestran que es importante contar con información que permita hacer planeación a mediano y largo plazo, ampliar e incluir estudios de temas relacionados, como la erosión eólica y otros tipos de degradación asociados al calentamiento global y estudios adicionales que contribuyan a completar el entendimiento de los cambios que se presentarán en el futuro y determinar acciones de previsión y cuidado del recurso suelo.

\section{Conclusiones e implicaciones}

El efecto de la disminución de precipitación pluvial estimada para el estado de Zacatecas, provocará una disminución en las pérdidas de suelo de las áreas de pastizal y de los principales tipos de vegetación en el estado de Zacatecas. Los valores medios de erosión hídrica son menores en las áreas de pastizal, seguida de las áreas de matorral, bosque y las más altas se presentan en el área de uso agrícola, aunque con menor impacto en estas dos últimas categorías. El cambio de uso del suelo hacía las actividades agrícolas puede conducir a pérdidas potenciales de suelo equivalentes a los valores medios. Se deben evaluar los efectos de la disminución de precipitación sobre las pérdidas de suelo por erosión eólica y otros tipos de degradación del suelo. 


\section{Literatura citada:}

1. IPCC. Climate Change 2014: Synthesis Report. Contribution of Working Groups I, II and III to the Fifth Assessment Report of the Intergovernmental Panel on Climate Change [Core Writing Team, RK Pachauri and LA Meyer editors]. IPCC, Geneva, Switzerland. 2014.

2. Echavarría CFG, Medina GG, Rumayor RAF, Serna PA, Salinas, GH, Bustamante WJG. Diagnóstico de los recursos naturales para la planeación de la intervención tecnológica y el ordenamiento ecológico. INIFAP. CIRNOC. Libro Técnico Nº10. 2009.

3. Soil Science Society of America. Glossary of soil terms. SSSA. Madison, WI. 53711. 1997.

4. UNCED. Earth summit agenda 21: Program of action for sustainable development. New York. United Nations Department of Public Information. 1992.

5. Comisión Nacional para el Conocimiento y Uso de la Biodiversidad. Quinto Informe Nacional de México ante el Convenio sobre la Diversidad Biológica (cdb). Conabio. México. 2014.

6. Secretaría de Medio Ambiente y Recursos Naturales. Inventario Estatal Forestal y de Suelos. Colección de inventarios forestales y de suelos. 2013-2014. México, DF. 2014.

7. INEGI (Instituto Nacional de Estadística y Geografía). Anuario estadístico y geográfico de Zacatecas. Aguascalientes, Ags. 2014.

8. INEGI. Conjunto de datos vectoriales. Edafología. Continuo Nacional. Escala 1:1000,000. 1998.

9. Renard KG, Foster GR, Weesies GA, McCool DK, Yoder DC. Predicting soil erosion by water: A guide to conservation planning with the Revised Universal Soil Loss Equation (RUSLE), Agricultural Handbook, 703. U.S. Government Printing Office, Washington, DC. 1997.

10. Eastman JR. Idrisi Selva manual. Versión 17. Clark labs Clark University. Worcester, Mass., USA. 2012.

11. Figueroa SB, Amante AO, Cortés HGT, Pimentel JL, Osuna ESC, Rodríguez JMO, Morales FJF. Manual de predicción de pérdidas de suelo por erosión. SARH Colegio de postgraduados. México, DF. 1991.

12. Ruiz-Corral JA, Medina-García G, Rodríguez-Moreno VM, Sánchez-González JJ, Villavicencio GR, Durán Puga N, Grageda GJ, et al. Regionalización del cambio climático en México Rev Mex Cienc Agríc Pub. Esp. Núm. 2013;13:2451-2464.

13. Walton D. Meyerson J. Neelin JD. Accessing, downloading, and viewing CMIP5 data. Earth System Grid Federation. 25 p. IPCC (Intergovernmental Panel on Climate Change). 2013. 
The physical science basis. Working group, I contribution to the fifth assessment report of the intergovernmental panel on climate change. Summary for policymakers. In: Stocker TF, et al editors. Switzerland. 2013.

14. INEGI. Conjuntos de datos de la Serie V de Uso del Suelo y Vegetación, escala 1:250 000. 2013.

15. SAS Institute Inc. SAS 9.3. Cary, NC: SAS Institute Inc. 2011.

16. Echavarría CFG, Serna PA, Bañuelos VR. Influencia del sistema de pastoreo con pequeños rumiantes en un agostadero del semiárido Zacatecano: II Cambios en el suelo. Téc Pecu Méx 2007;45(2):177-194.

17. Serna PA, Echavarría CFG. Caracterización hidrológica de un pastizal comunal excluido al pastoreo en Zacatecas, México. I. Pérdidas de suelo. Téc Pecu Méx 2002;40(1):37-53.

18. Echavarría CFG. Recurso suelo, clasificación, uso, degradación y disponibilidad. En: Mojarro DF, et al., editores. Agua subterránea en Zacatecas. Universidad Autónoma de Zacatecas. México, DF. 2013:97-133.

19. Eltaif NI, Gharaibeh MA. Aplicación de un modelo matemático para predecir y reducción de la erosión eólica en tierras áridas no protegidas. Revista Chapingo Serie Ciencias Forestales y del Ambiente, Volumen XVII, Edición Especial: 2011;195-206.

20. Aimar SB, Méndez MJ, Buschiazzo DE. Predicción de la erosión eólica potencial con el modelo EWEQ en dos suelos loesicos: efectos de las condiciones climáticas. CI. SUELO 2011;29(2):253-264.

21. Moore B, Allard G. Los impactos del cambio climático en la sanidad forestal. Organización de las Naciones Unidas para la Agricultura y la Alimentación. Departamento Forestal. FAO. 2009. 